\title{
Atención humanizada del embarazo: la mirada de gestantes que acuden a una unidad hospitalaria de salud ${ }^{1}$
}

\author{
María Mercedes Lafaurie Villamil ${ }^{2}$, María del Pilar Angarita de Botero ${ }^{3}$, Clara Inés Chilatra Guzmán ${ }^{4}$
}

Institución: Universidad El Bosque

\section{RESUMEN}

Abordar la humanización de la atención al embarazo desde la mirada de gestantes usuarias de una unidad hospitalaria pública de Bogotá, a partir de sus experiencias, preocupaciones y temores y propuestas. Estudio cualitativo en el cual participaron 20 gestantes atendidas por la unidad; la información se obtuvo con apoyo de un taller; se realizó un análisis temático de la información obtenida. Los embarazos son asumidos por las gestantes como "una bendición", a pesar de enfrentar, en ocasiones, situaciones adversas; los temores de las gestantes se centran especialmente en los dolores y complicaciones del parto y la posibilidad de morir o perder a sus hijos; se observan necesidades especiales en las gestantes adolescentes y las gestantes con alto riesgo obstétrico. También, las participantes proponen priorizar y agilizar la atención a las gestantes en los servicios, mejorar la información que reciben, garantizar el acompañamiento en el parto y fortalecer la escucha, la confianza y el buen trato hacia las usuarias. Según los hallazgos del estudio, el embarazo para el grupo de participantes es un evento positivo que, sin embargo, conlleva momentos de tensión, temores y preocupaciones, las cuales recaen, especialmente, en el devenir del parto. Dichas circunstancias pueden ser minimizadas, mediante la acción humanizada del equipo de salud, fortaleciendo su empoderamiento. Asimismo, se destacan los siguientes aspectos:: a nivel de calidad de la atención, la oportunidad e información hacia las usuarias y el acompañamiento durante el parto; a nivel relacional, responder a la demanda de calidez, escucha y confianza en el trato hacia las usuarias.

Palabras clave: Derechos-de-la-Mujer; Embarazo; Género; Investigación-Cualitativa; Parto-Humanizado.

DOI 10.15517/revenf.v0i38.38376

${ }^{1}$ Fecha de recepción : 17 de julio de 2019

2 Psicóloga. Universidad El Bosque. Colombia. Correo electrónico: lafauriemaria@unbosqure.edu.co

${ }^{3}$ Odontóloga. Subred Integrada de Servicios de Salud Norte. Colombia. Correo electrónico: piliangarita@yahoo.com

${ }^{4}$ Enfermera. Universidad El Bosque. Colombia. Correo electrónico: cchilatra@unbosque.edu.co 


\title{
Humanized pregnancy care: the look of pregnant women who go to a hospital health unit ${ }^{1}$
}

\author{
María Mercedes Lafaurie Villamil², María del Pilar Angarita de Botero ${ }^{3}$, Clara Inés Chilatra Guzmán ${ }^{4}$
}

Institution: El Bosque University

\begin{abstract}
To approach to humanization of pregnancy care from the perspective of pregnant users of a public hospital unit from Bogota, considering their experiences, concerns, fears and proposals. Qualitative study in which participated 20 pregnant women attended by the health services unit. It was implemented a workshop to obtain the information. A thematic analysis of the data was developed. Pregnant women assume pregnancies as "a blessing", despite having to face sometimes adverse situations; their fears was focused on the pains and complications of childbirth and the possibility of dying or losing their child; special needs are observed in adolescent pregnant women and in those with high obstetric risk. The participants propose to prioritize and speed up the attention to the pregnant women in the services, improve the information they receive, guarantee the accompaniment in the delivery and strengthen the listening, the trust and the good treatment to the users. According to the study's findings, pregnancy for the participants is a positive event that, however, involves moments of tension, fears and concerns, which fall especially on the upcoming of childbirth, which can be minimized by the health team's humanized action, strengthening their empowerment. In the level of quality of care, central aspects include opportunity in assistance, information to the users and accompaniment during childbirth. At the relational level, responding to the demand for warmth, listening and sureness in the treatment of users.
\end{abstract}

Keywords: Gender; Humanized-Delivery; Pregnancy; Qualitative-Research; Women's-Rights.

DOI 10.15517/revenf.v0i38.38376

${ }^{1}$ Reception date: July 17, 2019

Acceptance date: September 07, 2019

${ }^{2}$ Psychologist. El Bosque University. Colombia. E-mail: lafauriemaria@unbosqure.edu.co

${ }^{3}$ Dentist. Subnet Integrated Health Services North.Colombia. E-mail: piliangarita@yahoo.com

${ }^{4}$ Nurse. El Bosque University. E-mail: cchilatra@unbosque.edu.co 


\title{
Assistência humanizada à gravidez: o olhar de gestantes que frequentam uma unidade de saúde hospitalar ${ }^{1}$
}

\author{
María Mercedes Lafaurie Villamil², María del Pilar Angarita de Botero ${ }^{3}$, Clara Inés Chilatra Guzmán ${ }^{4}$
}

Instituição: Universidade El Bosque

\section{RESUMO}

Abordar a humanização do cuidado da gravidez na perspectiva de gestantes usuárias de uma unidade hospitalar pública de Bogotá, considerando suas experiências, preocupações, medos e propostas. Estudo qualitativo em que participaram 20 gestantes atendidas pela unidade; as informações foram obtidas com o apoio de um workshop; uma análise temática da informação obtida foi realizada. As gestações são assumidas pelas gestantes como "uma bênção", apesar de terem que enfrentar situações às vezes adversas; seus medos se concentram especialmente nas dores e complicações do parto e na possibilidade de morrer ou perder o filho; necessidades especiais são observadas em gestantes adolescentes e com alto risco obstétrico. Os participantes propõem priorizar e agilizar a atenção às gestantes nos serviços, melhorar as informações que recebem, garantir o acompanhamento na entrega e fortalecer a escuta, a confiança e o bom trato à mulheres usuárias. Segundo os achados do estudo, a gravidez para o grupo de participantes é um evento positivo que, no entanto, envolve momentos de tensão, medos e preocupações, que se enquadram principalmente no futuro do parto e podem ser minimizados pela ação humanizada da equipe de saúde, fortalecendo seu poder. Como aspectos a serem considerados, destacam-se: no nível da qualidade da assistência, a oportunidade, as informações aos usuários e o acompanhamento durante o parto; no nível relacional, responder à demanda de calor, escuta e confiança na maneira como as usuárias são tratadas.

Palavras-chave: Direitos-das-Mulheres; Gênero; Gravidez; Parto-Humanizado; Pesquisa-Qualitativa.

DOI 10.15517/revenf.v0i38.38376

${ }^{1}$ Data da recepção: 17 de Julho de 2019

Data da aceitação: 07 de Outubro de 2019

${ }^{2}$ Psicóloga. Universidade El Bosque. Colombia. Correio eletrônico: 1afauriemaria@unbosqure.edu.co

${ }^{3}$ Dentista. Serviços Integrados de Saúde em Sub-rede Norte. Colombia. Correio eletrônico: piliangarita@yahoo.com

${ }^{4}$ Enfermeira. Universidade El Bosque. Colombia. Correio eletrônico: cchilatra@unbosque.edu.co 


\section{INTRODUCCIÓN}

La Organización Mundial de la Salud (OMS) enfatiza en la importancia de mejorar las experiencias de las mujeres durante la gestación y el parto, mediante una atención digna y respetuosa y el derecho a no sufrir violencia o discriminación, lo cual, entre otras consecuencias, afecta la confianza de las mujeres en las instituciones y las desmotiva frente a la atención en salud ${ }^{1}$.

De manera relativamente reciente, en América Latina, se ha profundizado en el reconocimiento de la violencia obstétrica como una forma de la violencia de género que se expresa en una atención reproductiva autoritaria, en donde se desconoce la autonomía de las mujeres sobre sus cuerpos y, por tanto, pierden el protagonismo en un proceso propio ${ }^{2}$. En ese contexto, se homogeniza a las gestantes, por lo que se dejan de lado sus particularidades y sus necesidades; además de que se pueden llegar a ejercer sobre ellas malos tratos que afectan su dignidad como personas ${ }^{3}$.

Las mujeres en el proceso reproductivo pueden enfrentarse a temores, dolores e incomodidades que requieren ser comprendidos y resueltos ${ }^{4}$. En este sentido, reconocer la perspectiva de las mujeres y, en especial, las de aquellas pertenecientes a grupos sociales mayormente vulnerables, permite contar con referentes para la toma de decisiones con miras a avanzar hacia el parto humanizado. Dicha estrategia, basada en el consenso de expertos de la OMS, ha sido promovida en Colombia por el Ministerio de Salud y Protección Social ${ }^{5}$ y ha sido acogida por algunas unidades de atención hospitalaria del país. Cabe destacar que esta estrategia abarca todo el proceso reproductivo y considera esencial no solo el parto, sino también el periodo prenatal.

El parto humanizado busca enfrentar las deficiencias de los sistemas de salud en lo referente a la atención de la salud materna y mejorar la experiencia reproductiva de las mujeres, al tener en cuenta elementos como: la calidad, la equidad, el respeto a los derechos sexuales y reproductivos, la dignidad y la autonomía de las mujeres, el reconocimiento de sus particularidades y la minimización de procedimientos innecesarios, entre otros 5 . Asimismo, el proceso de humanización requiere responder a las necesidades de la mujer y a su apreciación individual de aquellos sentimientos y sensaciones que surgen frente a la expectativa del parto ${ }^{6}$.

Por tanto, con base en las recomendaciones propuestas por este modelo, relacionadas con la importancia de reconocer las necesidades de las gestantes y de minimizar sus temores frente al parto), el estudio realizado tiene como objetivo realizar una lectura, desde la humanización de la atención en salud materna, de la perspectiva de gestantes que acuden a una unidad hospitalaria de salud que implementa el modelo de parto humanizado en Bogotá; con el fin de allegar referentes para fortalecer, a nivel institucional, los procesos implicados en su desarrollo, bajo la luz de los lineamientos de Ministerio de salud y Protección social de Colombia ${ }^{5}$. Es así como, las experiencias en los embarazos, las preocupaciones y los temores relacionados con la gestación, el parto y el postparto fueron explorados a partir de un abordaje cualitativo, del mismo modo que las propuestas relacionadas con la atención en salud materna para fortalecer la humanización.

\section{MATERIALES Y MÉTODO}

En el estudio participaron 20 gestantes que acudían a una unidad de atención hospitalaria pública perteneciente a la Subred integrada de servicios de salud Norte E.S.E. de Bogotá en el primer semestre de 2018. El estudio 
realizado fue de tipo cualitativo con enfoque descriptivo e interpretativo, se partió de la premisa de que, si bien la investigación cualitativa es hermenéutica por definición, puede valerse, también, de la descripción como garantía de la validez de la investigación, lo cual da pie a lo denominado por Aguirre y Jaramillo como "epistemología realista".

Para efectos de este estudio, que está orientado a fortalecer aspectos inmersos en la política pública, el enfoque mencionado previamente provee la posibilidad de crear un hilo entre la subjetividad de las participantes y el ejercicio explicativo que exige la construcción de referentes con miras a fortalecer el modelo de humanización en el contexto específico de la unidad hospitalaria de salud.

Respecto a la información, esta se obtuvo a través de un taller lúdico para la construcción de expresiones narrativas. En este sentido, ese tuvo en cuenta el criterio de selección deliberada propuesto por MartínezSalgado $^{8}$; pues, se definió el tamaño de la muestra por criterio o por colección completa, tal cual lo describe la autora.

De igual modo, como criterios de inclusión, se estableció que las gestantes debían estar asistiendo al curso de preparación para la maternidad y la paternidad ofrecido por la unidad de atención hospitalaria vinculada al estudio. La edad menor a los 14 años actuó como criterio de exclusión. Por ende, todas las gestantes (39) que acudieron al curso y cumplían con los criterios de selección fueron invitadas a participar en el taller. Cabe mencionar que la convocatoria se realizó de manera previa, se les explicó a las mujeres el objetivo del estudio, el método utilizado y las particularidades del taller a desarrolladar.

Se logró la asistencia de cerca de la mitad de las invitadas, quienes fueron distribuidas en cuatro grupos de trabajo: dos de multigestantes y dos de primigestantes. Las participantes utilizaron seudónimos para firmar sus aportes y confirmaron su compromiso en participar mediante la gestión de un consentimiento informado; en el caso de las menores de edad, se utilizó asentimiento y consentimiento de sus progenitoras.

Relacionado con lo anterior, se consignaron, mediante un formulario diseñado para ello, datos generales sobre las participantes para su posterior caracterización. Asimismo, se implementó una estrategia de taller lúdico que les permitió a las usuarias dar a conocer por escrito sus experiencias y puntos de vista de manera libre, secreta y espontánea; pues, se conoce que existe aprehensión por parte de las usuarias frente a la posibilidad de expresar sus ideas críticas relacionadas con los servicios de salud ofrecidos para ellas en la unidad de atención hospitalaria.

El taller fue llevado a cabo en un salón privado destinado a actividades grupales del hospital y tuvo una duración de dos horas y media. Este fue orientado por dos investigadoras externas a la unidad de atención y contempló dos momentos: 1. Construcción narrativa autobiográfica "Mis experiencias con el embarazo" y 2. Construcción creativa con fines narrativos: se crearon de manera grupal personajes elaborados con material de reciclaje que representaran para las asistentes su identidad como gestantes. Estos personajes sirvieron de vehículo para consignar escritos de las mujeres sobre sus experiencias, temores, preocupaciones y propuestas. Cada personaje creado en grupo contó con: un baúl donde se consignaron las narraciones elaboradas por cada una de las gestantes sobre sus experiencias en el embarazo, a manera de pequeños libros; un cofre donde se almacenaron escritos sobre sus temores y sus preocupaciones frente al embarazo y parto; un portafolio donde se consignaron 
propuestas de cada una para fortalecer la humanización en los servicios de salud materna de la unidad hospitalaria.

Los textos escritos de las participantes fueron transcritos y la información fue analizada mediante el proceso de análisis temático propuesto por Mieles y colegas ${ }^{9}$ mediante el cual se identifica, se organiza, se analiza en detalle y se reportan patrones o temas a partir de una cuidadosa lectura y relectura de la información recogida. Las autoras mencionadas proponen la búsqueda de patrones recurrentes, que consisten en opiniones, percepciones, series de acontecimientos, experiencias o modos de vida que se repiten una y otra vez de maneras determinadas en contextos similares o diferentes. Este modelo analítico, si bien tiene una orientación fenomenológica, favorece la posibilidad de construir referentes descriptivos que expresan prioridades en cuanto al fortalecimiento del modelo de parto humanizado en el nivel institucional. Además, para fortalecer la confiabilidad del análisis, este se realizó por acuerdo entre investigadoras y, a su vez, los hallazgos se soportan en citas textuales y se interpretan a la luz de la literatura científica.

Cabe mencionar que el proyecto fue aprobado y cuenta con aval ético del Comité de investigación y proyección social de la Facultad de Enfermería de la Universidad El Bosque, según el Acta 0161 del 26 de enero de 2017. El estudio toma en cuenta los criterios éticos para las investigaciones médicas en seres humanos contemplados en la Declaración de Helsinki $2013^{10}$, por tanto, se garantizan la dignidad, la integridad, la autodeterminación, la privacidad y la confidencialidad a las participantes.

\section{RESULTADOS}

En este aparte, se presentará, en primer lugar, la caracterización de las participantes y, en segundo lugar, se describirán - a partir de sus expresiones y sus experiencias con el embarazo - tanto sus temores y sus preocupaciones más relevantes, como las principales propuestas que realizan para fortalecer la humanización en los servicios de atención materna en la unidad de salud a donde acuden.

\section{Caracterización del grupo de participantes}

Las 20 participantes fueron atendidas por una unidad hospitalaria de tipo público a la que suele tener acceso población de bajos ingresos económicos. En la Tabla No. 1 se presenta un consolidado de los datos sociodemográficos que permite tener una idea acerca del grupo vinculado al estudio. 
Tabla No. 1. Caracterización del grupo de participantes

\begin{tabular}{l|c|c}
\hline \multicolumn{1}{c|}{ Concepto } & Valores & Número de casos \\
\hline Edad & $14-18$ & 4 \\
& $19-23$ & 5 \\
& $24-28$ & 5 \\
& $29-33$ & 5 \\
& $34-38$ & 1 \\
\hline Edad gestacional & Primer trimestre & 1 \\
& Segundo trimestre & 13 \\
& Tercer trimestre & 6 \\
\hline Escolaridad & Primaria & 2 \\
& Secundaria incompleta & 6 \\
& Secundaria completa & 7 \\
& Técnico/ Tecnólogo & 3 \\
\hline Vive con pareja & Superior incompleta & 2 \\
\hline Paridad & Sí & 15 \\
& No & 5 \\
\hline
\end{tabular}

Fuente: la investigación

Entonces, se podría definir el perfil de las participantes como mujeres jóvenes en plena edad reproductiva, quienes, en tres cuartas partes de los casos, conviven con sus parejas. La Encuesta Nacional de Demografía y salud ENDS $2015^{11}$, estableció que solo la mitad de las mujeres colombianas entre 13 y 49 años (49.7\%) se hallan unidas, por lo que este grupo estaría por encima de lo esperado. Asimismo, de las 20 participantes, 12 han logrado un nivel de secundaria completa o más alto, lo cual resulta más bajo que el promedio nacional para el área urbana que es $88 \%$ según la ENDS. También,cuatro de las participantes son menores de 18 años y conviven con sus familias de origen; cabe destacar que en Colombia el 17.4\% de los primeros embarazos se dan en adolescentes, según la misma encuesta.

\section{La experiencia del embarazo}

Las experiencias con el embarazo fueron narradas por las participantes mediante relatos autobiográficos. A partir de la lectura de los textos, se construyeron las siguientes subcategorías, con base en los tópicos más recurrentes: "La maternidad como una bendición", "Dificultades en el embarazo juvenil", "Nuevos embarazos no planeados" y "Participación de la pareja como elemento positivo".

\section{La maternidad como una bendición}

De manera general, las gestantes se muestran satisfechas con sus embarazos, expresan que tener un hijo "es una bendición" y muestran una expectativa positiva frente a su maternidad, como se observa en esta narración de 
Silvia, primigestante: "Pues no fue planeado, es mi primer embarazo, pero aceptado y pues feliz, lo he tomado como una bendición de Dios".

\section{Dificultades en el embarazo juvenil}

Para las primigestantes, la idea de ser madres se reporta como altamente gratificante, aunque algunas-como Lucia y Salomé, ambas adolescentes--han atravesado dificultades, según lo que expresan en sus narraciones. Por un lado, Lucía relata que los primeros meses de su embarazo los pasó en medio de la angustia y la soledad, ya que enfrentó el rechazo de su pareja y se sintió incapaz de informar a su familia. No obstante, en su quinto mes de embarazo, luego de lograr el apoyo de sus padres, se presentó un cambio en su vivencia: "Todo fue cambiando, y pues ya me sentí mucho mejor (...) Ya quiero tener a mi hijo entre mis brazos; es lo mejor que me ha pasado es una experiencia hermosa. Me enamoré de absolutamente todo con mi hijo".

Por otro lado, Salomé tiene 14 años y describe de este modo su experiencia: "Sufro de cambios emocionales muy fuertes porque esto es duro para mi edad, me deprimo mucho; lloro mucho cada vez que pienso en los cambios que van a llegar a mi vida; pero a pesar de todo amo a mi bebé porque gracias a él comprendí lo que es la vida". Y añade lo siguiente : "No sé cómo lo voy a criar siendo tan pequeña cuando el bebé nazca".

\section{Nuevos embarazos no planeados}

Entre las multigestantes, también se observan relatos felices frente a la maternidad, aunque algunas de las participantes requirieron de un proceso de asimilación de su nueva condición, especialmente al tratarse de embarazos no planeados. Por ejemplo, Sandra tiene su tercer embarazo y, luego de haber tenido una cirugía en que le extirparon un ovario, para ella fue inesperado verse embarazada de nuevo, por lo que requirió de un proceso de adaptación a su nueva realidad: "Oh sorpresa, estaba en embarazo después de diez años, pero ya lo he asimilado y deseo conocerla y compartir con ella y mis otros bebés el amor inmenso que tengo para ellos, no soltarla nunca y estar siempre para ellos".

\section{Participación de la pareja como elemento positivo}

Poder contar con la pareja en el embarazo se destaca como elemento positivo entre algunas de las multigestantes, quienes en su primer embarazo tuvieron que enfrentarse solas a la maternidad. Esto relata Estrella: "Estoy en mi segundo embarazo y pues es mil por ciento diferente en todo. Comentaré a continuación: mi pareja ha estado presente desde hace cinco años conmigo y mi hija y ahora con este bebé fue una bendición porque él no podía tener bebés y pues estamos felices".

\section{Temores y preocupaciones}

En el análisis temático de los textos escritos por las participantes sobre sus temores y preocupaciones, se hallaron tópicos repetitivos que fueron organizados a partir de las siguientes subcategorías: "Temor al dolor y a posibles complicaciones en el parto", "Temor a la muerte propia o de su hijo", "Temor a que su hijo presente problemas de salud" y "Temores asociados a la vivencia emocional del parto". A continuación, se presentan los resultados más sobresalientes. 


\section{Temor al dolor y a posibles complicaciones en el parto}

El dolor en el parto y las complicaciones que puedan sobrevenir, como el parto pretérmino y requerir de una cesárea son aspectos que preocupan en general tanto a primigestantes, como a multigestantes. Por un lado, Ángela, primigestante, escribe lo siguiente: "Me da miedo a la hora del parto porque no sé cómo actuar si me duele o tan solo el susto no me deja pensarlo". Por otro lado, Valeria, multigestante, teme a la posibilidad de un parto prematuro: "Temor a que no llegue al periodo que tiene que llegar mi bebé". Finalmente, el temor a enfrentar una cesárea lo expresa de este modo Diana, primigestante: "Mi temor es no tener mi hijo en parto normal sino en cesárea, de saber cómo me va a ir, cómo me van a atender".

Con base en lo anterior, es notoria la preocupación de algunas gestantes con embarazos de alto riesgo a que se desencadenen complicaciones que puedan afectarlas a ellas y a sus hijos, lo que genera inseguridad frente al devenir del trabajo de parto y parto. En este sentido, Susana, multigestante expresa de este modo sus temores y preocupaciones: "Entrar en una crisis de epilepsia; entrar sin ninguna persona de confianza al trabajo de parto; riesgos que podamos correr mi hijo y yo si llegamos entrar a una cesárea. Miedo a entrar sin ninguna confianza al trabajo de parto".

\section{Temor a la muerte propia o de su hijo}

El temor a morir en el parto se presenta sobre todo entre las multigestantes, quienes se preocupan por la posibilidad de dejar a sus hijos abandonados. Isabela, multigestante lo expresa de este modo: "Temor a que me pueda pasar algo y dejar a mis hijos solos".

También, este temor se hace evidente entre quienes poseen problemáticas de salud que generan riesgos para el embarazo y parto, como Diana, multigestante, quien escribe lo siguiente: "En este embarazo tengo temor de que no resista, ya que he sentido muchas dolencias y que han dicho que es complicado ya que sufro del corazón".

El temor a perder a sus hijos por nacer, por sus problemas de salud, por su propia imprudencia o por fallas médicas, lo expresan algunas de las participantes; sin embargo, no se halló diferencia evidente entre las primigestantes y las multigestantes. En esta línea, Valentina, primigestante, teme que se presenten fallas médicas que puedan causar la muerte de su hijo: "El miedo de perder los bebés por ineficiencia de los doctores". Asimismo, dados sus problemas de salud, Violeta, multigestante, teme por su hijo: "Tengo muchísimo miedo de perder a mi bebé o que nazca con algún problema, ya que mi salud no está del todo bien”. De igual modo, esto le preocupa a Ángela, primigestante. "Hacer algo y sin querer perder el bebé y que al momento del parto no tenga ningún familiar que me acompañe”.

\section{Temor a que su hijo presente problemas de salud}

Se observa temor en las gestantes relacionado con la salud de sus hijos al nacer. Esto expone Lina, primigestante: "Temo que mi bebé nazca con bajo peso o que no tenga las vitaminas y nutrientes necesarios". En la misma línea, se presenta la intervención de Andrea, multigestante: "Temor a que vaya a salir con algún problema, no porque no se pueda enfrentar, sino porque hay rechazo de la gente". 


\section{Temores asociados a la vivencia emocional del parto}

La vivencia emocional durante el parto asociada a no contar con acompañamiento y la preocupación por la atención a ser recibida inquieta de manera especial a las gestantes. Por ejemplo, Zaida, multigestante, expresa su temor a no poder contar con la compañía de una persona significativa durante el parto: "Temo que al momento del parto no tenga ningún familiar que me acompañe".

También, Salomé muestra preocupación por el trato que pueda recibir durante el parto: "Mis temores son: cómo me voy a sentir el día del parto (...) cómo me van a tratar el día del parto los doctores".

\section{Propuestas para fortalecer la humanización}

Las propuestas de las participantes para fortalecer la humanización de la atención a las gestantes en la unidad hospitalaria se clasificaron a partir de dos amplias subcategorías: "Nivel calidad de la atención" y "Nivel relacional". A continuación se presentan los aspectos más destacados en cada nivel, acorde con su recurrencia:

\section{Nivel calidad de la atención}

En el nivel calidad de la atención, surgen cuatro tópicos a partir de las propuestas de las participantes para el fortalecimiento de la humanización en la institución, que son: "Oportunidad de la atención", "Pertinencia y claridad de la información a las usuarias", "Priorización de la atención a las gestantes" y "Garantía del acompañamiento durante el parto". Las particularidades de cada uno de ellos se especifican a continuación:

a) Oportunidad de la atención

Recibir atención oportuna y eliminar la tramitología son temas recurrentes en las propuestas de las participantes, quienes son reiterativas al mencionar la necesidad de minimizar los tiempos de espera que en ocasiones deben enfrentar. Esto expresa Milena, primigestante: "La atención de mi embarazo en el hospital ha sido buena, me han atendido bien (...) Mi única recomendación es que al momento de hacer exámenes de laboratorio no tengamos que esperar tanto y más cuando estamos en ayunas".

b) Pertinencia y claridad de la información a las usuarias

Recibir información pertinente y clara sobre los procedimientos administrativos es otra de las recomendaciones destacadas, como lo expone Diana, multigestante: "hay que dar mejores indicaciones de qué es lo que hay que hacer".

c) Priorización de la atención a las gestantes

Las participantes destacan entre sus propuestas la atención diferencial y prioritaria a las gestantes, tal como lo hace Carmen, una de las multigestantes: "La atención general en las instituciones debería ser mucho más óptima y prioritaria para las mamitas". 
d) Garantía del acompañamiento durante el parto

El contar con acompañamiento durante el trabajo de parto y parto es otro aspecto que se señala, como lo hace Johana: "Que el día del parto nos pongan atención y nos acompañe una enfermera o un familiar".

\section{Nivel relacional}

En el nivel relacional, surgen tres tópicos recurrentes: "Satisfacción con la atención”, "Recibir más comprensión y trato más cálido", "Sentirse en confianza y ser escuchadas". Se detallan a continuación:

\section{a) Satisfacción con la atención}

En general, las gestantes se muestran satisfechas con la atención recibida y reconocen los cambios favorables que se han venido dando con el tiempo en la unidad de atención hospitalaria, como se puede apreciar en la intervención de Lucero, multigestante: "A diferencia de mi primer embarazo, el trato médico ha sido mucho mejor; me han tratado bien los médicos de todas las especialidades".

b) Recibir más comprensión y trato más cálido

Recibir un trato más cálido por parte de algunos miembros de equipo de salud es parte de las necesidades de las participantes. Carmen, multigestante, lo expresa de esta manera: "Que nos entiendan bien y nos traten bien en todas las citas médicas".

\section{c) Sentirse en confianza y ser escuchadas}

Ser más escuchadas, así como poder sentirse más en confianza, son también parte de las necesidades expresadas por las participantes, como lo resume Johana, primigestante en su propuesta: “Que en la atención sean más humanistas, pues como embarazadas estamos más sensibles y queremos sentirnos más cómodas y en confianza. Que te brinden ese confort que a uno le transmite esa confianza de poder preguntar tantas cosas".

\section{DISCUSIÓN}

Si bien la aceptación del embarazo como "una bendición" fue el común denominador de las experiencias narradas por las participantes, la falta de apoyo psicosocial, el enfrentar un embarazo a temprana edad y vivir un embarazo inesperado, crearon situaciones adversas para algunas de las gestantes; no obstante; varias de estas situaciones fueron resolviéndose con el paso del tiempo. Similares resultados hallaron Cáceres y colegas ${ }^{12}$, a partir de una investigación cualitativa sobre la maternidad vista desde la perspectiva de un grupo de gestantes, en donde la maternidad, en los discursos de las entrevistadas, surge como una experiencia positiva, aunque con diferentes matices relacionados estrechamente con el acompañamiento que reciben de su entorno afectivo. Según lo exponen las autoras, la maternidad como fenómeno sociocultural complejo puede darse en un ambiente favorable, de soporte a la gestante o puede darse en un ambiente adverso de vulnerabilidad económica, baja escolaridad y barreras de acceso a los servicios de salud, lo cual puede significar la diferencia. 
Entre los temores y preocupaciones asociadas al proceso reproductivo, aquellos que se relacionan con el parto resultan ser el punto de quiebre, según lo que se concluye a partir de las intervenciones de las participantes. El parto, a diferencia del embarazo que por su proceso paulatino facilita la adaptación gradual de las transiciones, provoca cambios repentinos y fuertes como la intensidad del dolor y la imprevisibilidad, lo cual crea en las mujeres situaciones de ansiedad, sufrimiento e inseguridad ${ }^{13}$.

En el caso de las mujeres que participaron en el estudio, el posible desenvolvimiento del embarazo causa especial aprehensión por varias razones que resultan recurrentes: el enfrentamiento con el dolor, la posibilidad de que se presenten complicaciones - incluso las que deriven en la muerte propia o de su hijo-, la posibilidad de no recibir un buen trato o atención adecuada y el no poder contar con acompañamiento durante el proceso. En este sentido, Schmid $^{14}$ afirma que, detrás del miedo a lo que les sucederá durante el parto y al dolor, puede encontrarse en las mujeres necesidades que podrían verse resueltas mediante el respeto, la claridad frente a los procedimientos de la institución hospitalaria y un acompañamiento continuo y acogedor.

Asimismo, Spanó y colegas ${ }^{15}$, en un estudio con primigestantes, halló como temores recurrentes la muerte propia y la del hijo y-como algo inherente al parto-, el temor al dolor, por lo que sugieren reconocer como acciones de cuidado empoderar a las gestantes para asumir las riendas del proceso de parto en mayor medida.

De acuerdo con los hallazgos obtenidos, se destaca la necesidad especial que tienen las gestantes de alto riesgo por minimizar las preocupaciones y los temores asociados con la posibilidad de que su situación de salud incida negativamente en el desenlace del embarazo, del parto y en las condiciones del niño por nacer. La oportunidad de intercambiar experiencias, de expresar libremente los sentimientos y de reflexionar con el profesional de salud acerca el embarazo de alto riesgo y las situaciones que lo involucran, ayudan a las gestantes a enfrentar de mejor manera esta condición, en la que estas deben ocupar un lugar destacado ${ }^{16}$.

Además, vencer el miedo al parto y ofrecer a las gestantes la posibilidad de discutir sus dudas y hacer preguntas en un ambiente en que la discusión se facilite, ya sea a nivel individual o grupal, forma parte de las condiciones para garantizar un parto humanizado ${ }^{5}$. Además el temor frente al parto es determinante en la presentación de cesáreas y se relaciona ampliamente con las experiencias desfavorables de las mujeres ${ }^{17}$; por lo tanto, responder a las inquietudes y emociones de las gestantes por parte del personal de salud reduce temores e incrementa la confianza en ellas ${ }^{18}$.

De la misma forma, algunas participantes temen que sus hijos presenten problemas de salud y, en ciertos casos, especialmente entre las más jóvenes, sus temores se orientan hacia ser incapaces de cumplir adecuadamente el rol materno. En esta línea, Cáceres y sus colegas ${ }^{12}$ señalan que la maternidad conlleva una preocupación especial relacionada con el cuidado del niño, con la falta de preparación para la crianza, con la falta de autonomía y con la dependencia económica; además, señalan los temores relacionados con circunstancias como la posibilidad de malformaciones congénitas en el niño y razones relacionadas con la condición laboral y con no ser buenas madres, lo cual se podría minimizar con acceso a servicios de salud humanizados.

También, entre las prioridades expuestas por las participantes para fortalecer la humanización de los servicios, se encuentran: la atención oportuna, la minimización de la tramitología y la información clara y pertinente a las gestantes. En la misma línea, en un estudio realizado por Pintado y colegas ${ }^{19}$ en Oaxaca, México sobre la atención 
materna desde la valoración de las usuarias, se encontró que entre los aspectos que le restan calidad al control prenatal se destacaron los largos tiempos de espera y la necesidad de regresar para la toma de muestras o estudios.

En el nivel relacional, es imperativo para las gestantes, ser escuchadas, encontrar entornos amigables donde puedan recibir respuesta a sus inquietudes y ser tratadas siempre de manera amable y respetuosa. Por tanto, en el cuidado humanizado de la salud materna, la formación del personal de salud en asuntos de género suscita cambios relacionales y lleva a empoderar a las mujeres para el ejercicio consciente de sus derechos ${ }^{20}$.

En línea con lo anterior, tal como lo contempla la $\mathrm{OMS}^{21}$, se requiere hacer de los derechos humanos, de la equidad de género y de la ética, los principios clave; así como, del cuidado holístico centrado en las personas, el modo de aproximación en la salud sexual y reproductiva. Relacionado con ello, Cáceres y Nieves ${ }^{4}$ sugieren generar procesos de reflexión, autocrítica y retroalimentación para lograr reducir inequidades de las que suelen ser objeto las gestantes más vulnerables por su edad, su marginalidad o su situación de riesgo, y, así, ofrecer una atención humanizada diferencial y centrada en las necesidades de cada gestante.

Finalmente, cabe destacar que, por ser un estudio cualitativo, la limitación del presente trabajo radica en que sus hallazgos no pueden ser generalizables a grupos humanos diferentes del que fue abordado; por tanto, su lectura requiere contemplar el peso del contexto social y cultural que envuelve las vidas de las participantes.

\section{CONCLUSIONES}

Según los hallazgos del estudio, el embarazo para el grupo de participantes es un evento positivo que, sin embargo, posee momentos de tensión, temores y preocupaciones, que recaen especialmente en el devenir del parto, lo cual podría minimizarse mediante la acción humanizada del equipo de salud. Asimismo, es imperioso reconocer a las adolescentes y gestantes con embarazos de alto riesgo como grupos particularmente vulnerables; así como, incluir a aquellas con carencia de apoyo psicosocial.

Además, más allá de las condiciones sociales y de las circunstancias de sus usuarias, el equipo de salud debe diseñar procesos de atención y de acompañamiento psicosocial que fortalezcan la confianza de las gestantes en sí mismas y en la institución.

Del mismo modo, como aspectos críticos y susceptibles por ajustarse para consolidar, desde sus diferentes dimensiones, el modelo de parto humanizado en la unidad hospitalaria, se destacan: la importancia de cualificar la atención en cuanto a oportunidad e información a las usuarias, al reconocer el buen trato como algo central y la escucha de las inquietudes y la respuesta a las necesidades. En este sentido, se sugiere seguir consolidando el modelo de parto humanizado desde la dimensión relacional, mediante procesos de reflexión en los que se involucre tanto al personal de salud, como al personal administrativo y se enfatice en la formación en género y en derechos, lo que permitiría profundizar en aspectos como las desigualdades que afectan a las mujeres en los procesos reproductivos. En la misma línea, la reflexión al interior de institución en torno a la violencia obstétrica y a los modos de erradicarla, puede generar logros significativos. 
El parto humanizado, como modelo orientador de la acción, implica un proceso continuo que se alimenta especialmente de la apertura al cambio, la formación, la reflexividad del equipo de trabajo, la evidencia científica y el diálogo permanente con las usuarias y sus familias. Por ende, llevar a la gestante a ser la protagonista del proceso reproductivo, debe convertirse en un reto para el equipo de salud materna.

\section{Declaración de conflicto de intereses}

Las autoras declaran que no presentan ningún conflicto de intereses.

\section{REFERENCIAS BIBLIOGRÁFICAS}

1. World Health Organization. The prevention and elimination of disrespect and abuse during facility-based childbirth. Washington: Department of Reproductive Health and Research, World Health Organization; 2014. en: https://apps.who.int/iris/bitstream/handle/10665/134588/WHO_RHR_14.23_eng.pdf?sequence=1

2. Almonte G. Violencia ginecológica y obstétrica. Revista Décsir.EAM. 2016; (2):1-10. Disponible en: http://decsir.com.mx/wp-content/uploads/2016/03/1-indagaciones.pdf

3. Castrillo MB. Dime quién lo define y te diré si es violento. Sexualidad, salud y sociedad. 2016; (24): 43-68. DOI: http://dx.doi.org/10.1590/1984-6487.sess.2016.24.03.a

4. Cáceres-Manrique FM, Nieves-Cuervo FM. Atención humanizada del parto. Diferencial según condición clínica y social de la materna. Rev Col Obstet Ginecol. 2017; 68(2): 128-134. DOI: http://dx.doi.org/10.18597/rcog.3022

5. República de Colombia. Ministerio de Salud y Protección Social. Guías de atención Integral para la prevención, detección temprana y tratamiento de las complicaciones del embarazo, parto o puerperio para uso de profesionales de la $2013 . \quad$ Dalud. 2 Disponible https://www.minsalud.gov.co/sites/rid/Lists/BibliotecaDigital/RIDE/INEC/IETS/G.Corta.Embarazo.y.parto.P rof. Salud.2013\%20(1).pdf

6. Borges-Damas L, Sánchez-Machado R, Domínguez-Hernández R, Sixto-Pérez A. El parto humanizado como necesidad para la atención integral a la mujer. Revista Cubana de Obstetricia y Ginecología. 2019; 44(3). Disponible en: http://revginecobstetricia.sld.cu/index.php/gin/article/view/226

7. Aguirre, J.C. y Jaramillo, J.G. El papel de la descripción en la investigación cualitativa Cinta moebio. 2015; 53: 175-189. Disponible en: file://D:/User/Downloads/37207-1-128088-1-10-20150827.pdf

8. Martínez-Salgado C. El muestreo en investigación cualitativa. Principios básicos y algunas controversias. Ciênc. Saúde Coletiva; 2012; 17(3): 613-619. DOI: http://dx.doi.org/10.1590/S1413-81232012000300006 
9. Mieles Barrera MD, Tonon MG, Alvarado SV. Investigación cualitativa: el análisis temático para el tratamiento de la información desde el enfoque de la fenomenología social. Universitas humanística. 2012; (74): 195 - 225. Disponible en: http://www.scielo.org.co/pdf/unih/n74/n74a10.pdf

10. Asociación Médica Mundial. Declaración de Helsinki de la AMM. Principios éticos para las investigaciones médicas en seres humanos. 2013. Disponible en: http:/www.isciii.es/ISCIII/es/contenidos/fdinvestigacion/fd-evaluacion/fd-evaluacion-etica-investigacion/Declaracion-Helsinki-2013-Esp.pdf

11. Profamilia. Encuesta Nacional de Demografía y Salud 2015. Bogotá: Profamilia; 2015. Disponible en: https://profamilia.org.co/investigaciones/ends/

12. Cáceres-Manrique FM, Molina-Marín G, Ruiz-Rodríguez M. Maternidad: un proceso con distintos matices y construcción de vínculos. Aquichan; 2014; 14(3): 316-326. DOI: http://dx.doi.org/10.5294/aqui.2014.14.3.4

13. Da Rocha Pereira R, Franco SC, Baldin N. El dolor y el protagonismo de la Mujer en el parto. Rev Bras Anestesiol. 2011; 61(3): 204-210. Disponible en: http://www.scielo.br/pdf/rba/v61n3/es_v61n3a14.pdf

14. Schmid V. El dolor del parto. Una nueva interpretación de la fisiología y la función del dolor ( $2^{\mathrm{a}}$ ed.). Tenerife, España: Ob Stare; 2012.

15. Spano A, Jorge C, Almeida A, Gomes F. Childbirth experence according to a group of Brazilian primíparas. Midwifery. 2012; 28(6): e844-9. DOI: https://doi.org/10.1016/j.midw.2011.09.014

16. Amorim TV, Souza Ívis E de O, Moura MAV, Queiroz ABA, Salimena AMO. Perspectivas de los cuidados de enfermería en el embarazo de alto riesgo: revisión integradora. Enf Global. 2017; 16(2): 500-543. DOI: http://dx.doi.org/10.6018/eglobal.16.2.238861

17. Kordi M, Bakhshi M, Masoud S, Esmaily H. Effect of a Childbirth Psychoeducation Program on the Level of Fear of Childbirth in Primigravid Women. Evidence Based Care Journal. 2017; 7 (3): 26-34. DOI: http://dx.doi.org/10.22038/EBCJ.2017.25676.1575

18. Mohamamdiriz S, Mohamadirizi M, Mohamadirizi S. The comparison of fear of childbirth and sense of coherence among low-risk and high-risk pregnancy women. J Educ Health Promot. 2018; 7: 143. DOI: http://dx.doi.org/10.4103/jehp.jehp_179_17

19. Pintado A, Lastra Z, Sachse M. Atención materna durante el embarazo, parto y puerperio en unidades de primer nivel en Oaxaca, México. Medicina social. 2014; 9(1): 13-20. Disponible en: http://www.medicinasocial.info/index.php/medicinasocial/article/view/775/1540

20. Lafaurie MM, Antolinez P. Una mirada de género al embarazo después de los 35 años: experiencias de mujeres atendidas por la red de salud de Bogotá D.C. Rev. Col. Enfer. 2014; 9(9): 95-107. DOI: http://dx.doi.org/10.18270/rce.v9i9.569 


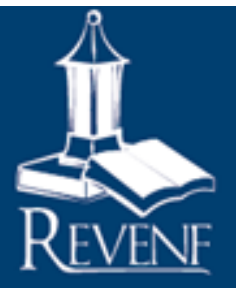

21. World Health Organization. WHO Consolidated Guideline on self-care interventions for health. Sexual and reproductive health and rights. Geneva: World Health Organization; 2019. Disponible en: https://www.who.int/reproductivehealth/publications/self-care-interventions/en/ 